weibliche Beschäftigte) sich wegen psychischer Störungen stationär behandeln lassen mussten. Dabei liegen alle Menschen, die in der Pflege arbeiten, ohnehin schon jeweils mehr als 55 Prozent über dem Schnitt der Krankenhaustage aller Berufstätigen, die aufgrund psychischer Diagnosen im Krankenhaus waren.

Bei der stationären Versorgung sind es nicht nur die Beschäftigten der Heime mit den meisten Krankenhaustagen, auch die regionalen Unterschiede sind hier am größten. Besonders viele Krankenhaustage gab es im Saarland, rund ein Drittel weniger waren es in Sachsen.

\section{Betriebliche Gesundheitsförderung ausbaufähig}

Bei einer Umfrage unter 2.000 Beschäftigten betonten neun von zehn Befragten: Die Förderung von Gesundheit an ihrem Arbeitsplatz ist ihnen wichtig oder sehr wichtig. Allerdings geben weniger als die Hälfte an, dass es überhaupt solche Maßnahmen in ihrem Unternehmen gibt. Es zeigt sich durchaus ein Zusammenhang: Teilnehmer an betrieblicher Gesundheitsförderung leben laut eigenen Angaben auch außerhalb ihres Berufs aktiver und haben größere Zuversicht, ihren Beruf auch längerfristig ausüben zu können.

\section{Mehr Gesundheitsförderung in der Altenpflege}

Von diesen Erkenntnissen sollte auch die Altenpflege profitieren, allerdings gibt es hier mehrheitlich (57 Prozent) (noch) keine betriebliche Gesundheitsförderung (BGF). Sind solche Maßnahmen jedoch vorhanden, dann ist die Inanspruchnahme dort sehr hoch (79 Prozent).

Dass sich betriebliche Gesundheitsförderung lohnt und auch Fehlzeiten reduziert, konnte in zahlreichen weiteren Studien gezeigt werden. Das zahlt sich auch für Arbeitgeber aus: Durchschnittlich werden - konservativ gerechnet für jeden in die betriebliche Gesundheitsförderung investierten Euro allein durch die Reduktion von Fehlzeiten 2,70 Euro eingespart.

\section{NACHRICHTEN}

\section{Fischbach ist neue Pflegebevollmächtigte}

Ingrid Fischbach, bisher Staatssekretärin im Bundesgesundheitsministerium, ist neue Patientenbeauftragte und Pflegebevollmächtigte der Bundesregierung. Die CDU-Politikerin Fischbach löst den bisherigen Amtsinhaber Karl-Josef Laumann ab, der in die Landesregierung von Nordrhein-Westfalen gewechselt ist. Dort hat der Unions-Politiker das Ressort für Arbeit, Gesundheit und Soziales übernommen.

Ingrid Fischbach ist ursprünglich Lehrerin für die Fächer Deutsch und Geschichte. Zuletzt unterrichtete sie an einer Gesamtschule in Herne. Seit 1998 sitzt Fischbach für die CDU im Bundestag. Seit 2013 ist sie Parlamentarische Staatssekretärin im Bundesgesundheitsministerium.

Ihrem Vorgänger Laumann dankte Bundesgesundheitsminister Hermann Gröhe für seine "herausragende Arbeit". Zusammen habe man in den letzten Jahren „viele Verbesserungen für Patienten und Pflegebedürftige erreicht". Dabei

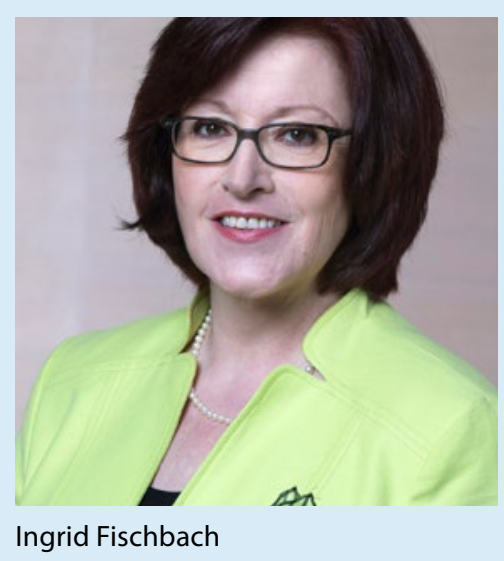

verwies der Gesundheitsminister insbesondere auf die Umsetzung des neuen Pflegebedürftigkeitsbegriffs und die Heil- und Hilfsmittelversorgung.

www.patientenbeauftragte.de

\title{
DKI-Gutachten zur Intensivpflege
}

Die Einführung von Pflegepersonaluntergrenzen in den pflegesensitiven Bereichen unserer Krankenhäuser ist ein entscheidender Schritt für die Sicherung des allgemeinen Pflegebedarfs. Damit muss sich auch die Deutsche Krankenhausgesellschaft endlich ihrer Verantwortung für eine ausreichende und zugleich hoch qualifizierte Personalbesetzung in der Pflege stellen. Das hat der Deutsche Pflegerat (DPR) in Berlin vor der Presse deutlich gemacht.

Mittelfristig fordert der DPR die Festlegung einer Personalmindestbesetzung für die Pflege in allen Krankenhausbereichen, die dennoch deren notwendige personelle Flexibilität gewährleistet. Ein Plädoyer für mehr Personal in der Pflege hilft uns nicht weiter. Das hat uns die Vergangenheit deutlich gezeigt. Hier braucht es Fakten, die der Gesetzgeber zu Recht geschaffen hat und die es jetzt gilt, zügig umzusetzen.

Anlässlich der Vorstellung und Bewertung des Gutachtens „,Personalsituation in der Intensivpflege und Intensivmedizin" des Deutschen Krankenhausinstituts (DKI) kommentierte der DPR weiter:
Die pflegerische Versorgung der Patienten in der Intensivpflege ist vielleicht objektiv gut, wie die Deutsche Krankenhausgesellschaft mitteilt, faktisch reibt man sich jedoch verwundert die Augen. Nach wie vor fehlen in unseren Krankenhäusern zehntausende Mitarbeiterinnen und Mitarbeiter in der Pflege. Zahlreiche aktuelle Studien zeigen, mit welchen enormen und stetig steigenden Arbeitsbelastungen die professionell Pflegenden umzugehen haben. Die Folgen sind enormer Stress sowie körperliche und psychische Belastungen bis hin zur Flucht aus dem Beruf. Die Personalsituation der Pflege im Krankenhaus ist weiterhin bedrohlich.

Allein vor diesem Hintergrund sind die im DKI-Gutachten genannten Hinweise auf eine verbesserte pflegerische Versorgung in der Intensivpflege allenfalls als Schritt dahingehend zu werten, dass sich eine schlechte Situation etwas verbessert hat, mehr jedoch nicht. Die Zeit des Schönredens und Beschwichtigens muss endgültig vorbei sein.

www.deutscher-pflegerat.de 